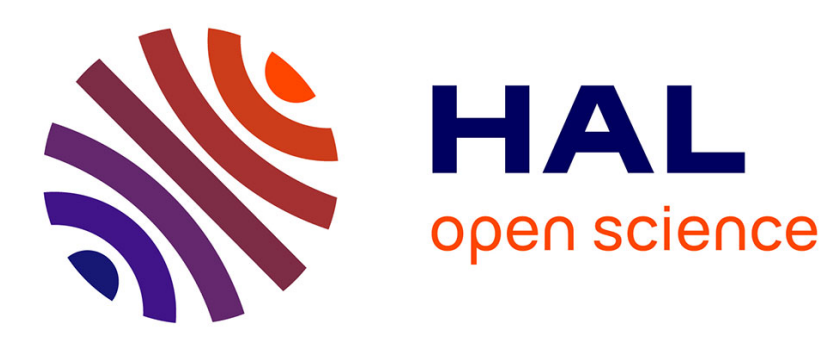

\title{
Daniel Halévy et le mouvement ouvrier. Libéralisme, christianisme social et socialisme
}

\author{
Sebastien Laurent
}

\section{To cite this version:}

Sebastien Laurent. Daniel Halévy et le mouvement ouvrier. Libéralisme, christianisme social et socialisme. Mil neuf cent. Revue d'histoire intellectuelle, 1999, 17 (1), pp.7-25. 10.3406/mcm.1999.1200 . hal-03176840v2

\author{
HAL Id: hal-03176840 \\ https://hal.science/hal-03176840v2
}

Submitted on 24 Mar 2021

HAL is a multi-disciplinary open access archive for the deposit and dissemination of scientific research documents, whether they are published or not. The documents may come from teaching and research institutions in France or abroad, or from public or private research centers.
L'archive ouverte pluridisciplinaire $\mathbf{H A L}$, est destinée au dépôt et à la diffusion de documents scientifiques de niveau recherche, publiés ou non, émanant des établissements d'enseignement et de recherche français ou étrangers, des laboratoires publics ou privés.

\section{(ㅇ)(1) $\$$}

Distributed under a Creative Commons Attribution - NonCommercial - NoDerivatives| 4.0 


\section{Daniel Halévy \\ et le mouvement ouvrier \\ Libéralisme, christianisme social et socialisme *}

C'est dans le ghetto bavarois de Fürth à la fin du XVIII ${ }^{\mathrm{e}}$ siècle que la famille Halévy trouve son origine. Jaakov Lévi quitta cette terre avec son fils Élie (1760-1826) pour gagner la France au moment de la Révolution française. L'intégration d'Élie - cantor à la synagogue de la rue des Victoires - à la communauté juive fut presque aussi rapide que celle de son fils Léon (1802-1883) à la société parisienne. Celui-ci formé au lycée sous la Restauration et non à l'école consistoriale, devint le secrétaire de Saint-Simon à la fin de sa vie. Enseignant, polygraphe, il fut l'un des premiers concepteurs du franco-judaïsme, vecteur d'intégration de la communauté juive. Dans le Résumé de l'histoire des juifs modernes qu'il écrivit en 1828, il appelait ses coreligionnaires à une fusion avec la population française. Léon ayant épousé une catholique, c'est à la deuxième génération que le détachement à l'égard de la religion juive commença à se réaliser. C'est donc dans une famille où la judaïcité se perdait que son fils Ludovic (1834-1908), baptisé dans la foi catholique, fut élevé. Simple rédacteur au secrétariat du ministère d'État, c'est en prêtant sa plume au duc de Morny qu'il trouva une protection

${ }^{*}$ Cf. notre thèse de doctorat: Daniel Halévy (1872-1962), une écriture entre littérature et politique. Du libéralisme au traditionalisme, sous la dir. de Serge Berstein, Paris, IEP, 1999, soutenue en janvier 2000. 
et un succès littéraire rapide dans l'opérette ${ }^{1}$, genre nouveau s'imposant sur la scène dramatique. Son demi-frère Anatole Prévost-Paradol (1829-1870), de sensibilité libérale et orléaniste, fut un des plus jeunes collaborateurs du Journal des Débats $^{2}$. Dès le début des années 1860 , il devint l'un des plus actifs soutiens de l'opposition libérale dont il synthétisa le programme dans La France nouvelle (1868). Ludovic partageait la sensibilité libérale de Paradol avec un attachement supplémentaire à la dynastie d'Orléans. Proche de l'entourage orléaniste du prince, Eugène Dufeuille, Albert de Broglie ou encore Joseph Othenin d'Haussonville, la sensibilité idéologique de Ludovic était marquée par un libéralisme conservateur, hostile à la démocratie ${ }^{3}$. La réussite littéraire et l'évolution sociale de Ludovic Halévy montrent que la famille sut utiliser tout au long du siècle et de façon efficace les possibilités d'intégration et d'ascension sociale offertes par la société démocratique postrévolutionnaire.

\section{La maturation d'une pensée sociale}

Élie Halévy (1870-1937) et son frère Daniel (1872-1962) reçurent tous deux la marque libérale familiale ${ }^{4}$ ainsi qu'une éducation religieuse issue de la famille maternelle protestante. L'aîné se tourna vers la philosophie alors que Daniel hésita longuement, accomplissant sans conviction des études d'arabe qui masquaient à peine un dilettantisme profond. Dès l'époque du lycée Condorcet il fonda avec ses camarades des petites revues littéraires, ébauches du Banquet, publié de 1892 à 1893. En fait, un souci social était apparu assez tôt, à l'âge de 17 ans, alors qu'il achevait sa classe d'humanités. Ce qu'il

1. Cf. Eric C. Hansen, Ludovic Halévy : A Study of Frivolity and Fatalism in Nineteenth Century France, Boston-Londres, University Press of America, 1987.

2. Cf. Pierre Guiral, Prévost-Paradol 1829-1870. Pensée et action d'un libéral sous le Second Empire, Paris, PUF, 1955.

3. Cf. Ludovic Halévy, Carnets, Paris, Calmann-Lévy, 1935, t. 1 : 1862-1869, et t. $2: 1869-1870$, introductions et notes de Daniel Halévy.

4. Nous renvoyons à notre étude: "Élie et Daniel Halévy au miroir du libéralisme français ", in Élie Halévy et l'ère des tyrannies, colloque international tenu à Rome en novembre 1998 (à paraître en éd. bilingue), ainsi qu'aux contributions de Vincent Duclert et de Nicolas Baverez. 
appelait alors « socialisme ${ }^{5}$ n'était rien d'autre qu'un sentiment idéaliste, celui d'un droit égal pour tous au «bonheur ». C'est par des lectures sociales postérieures marquées par un fort éclectisme, que les contours de ce sentiment se transformèrent peu à peu en une pensée socialiste. Il se lança en effet dans la lecture d'une série d'ouvrages relevant de quatre nuances idéologiques diverses : le socialisme français proudhonien, le renouveau spiritualiste protestant, les socialismes allemands et la sociologie libérale anglaise. En Proudhon, il distinguait un moraliste et un juriste plus qu'un idéologue, et déplorait l'économisme de certains de ces ouvrages. Lecteur du Capital, de Capital et travail de Ferdinand Lassalle (18251864) ainsi que de La quintessence du socialisme d'Albert Schaeffle (1831-1903), il approcha ainsi la diversité du socialisme allemand. The Study of Sociology d'Herbert Spencer (1820-1903), analyse historiciste du déclin de l'État, rejoignait Proudhon dans certaines de ses conclusions. Cependant, la découverte des Études sociales du philosophe protestant Charles Secrétan (1815-1895) eut assurément une importance majeure dans la formation de sa pensée sociale ${ }^{6}$. Dans cet ouvrage, le philosophe suisse affirmait son hostilité au socialisme collectiviste comme au capitalisme libéral. La recherche d'une voie médiane incitait l'auteur à adopter une analyse réformiste reposant sur le système coopératif, la participation aux bénéfices et une réforme «morale » de l'ouvrier ${ }^{7}$. Daniel Halévy avait tracé en 1890 le plan d'un ouvrage qu'il souhaitait consacrer au «Socialisme idéaliste». La première partie du livre était une étude comparée du socialisme allemand «théorique » et du socialisme français «pratique » (Louis Blanc, Proudhon). Il envisageait ensuite d'étudier Dieu, la morale religieuse, le but final du socialisme idéaliste étant de réaliser la «cité de l'amour» et la «cité de Dieu " ${ }^{8}$. La marque de Secrétan sur cette première formulation de la pensée sociale d'Halévy adolescent apparaît très nettement.

5. Archives privées Jean-Pierre Halévy (APH), Journal, 2729 mai 1889.

6. Les réflexions de D. Halévy consignées dans des volumes de Notes sont émaillées d'analyses et de citations de Secrétan.

7. Cf. Charles Secrétan, Etudes sociales, Lausanne, F. Payot Ed., 1889.

8. APH, Journal, 25 décembre 1890. 
La dimension idéaliste de cette formulation ne trouve pas seulement son origine dans la sensibilité protestante de Daniel Halévy : parallèlement à ces lectures, il découvrit la misère des quartiers ouvriers de la capitale, Saint-Ouen, Clignancourt, la Grande-Carrière, les Épinettes. Il franchit une première étape sur la voie du militantisme dès 1890 en s'abonnant à la Revue socialiste et en allant signer la pétition du $1^{\mathrm{er}}$ mai à la Bourse du travail. Il fut également sollicité par des œuvres philanthropiques protestantes et fut convaincu dès lors de l'importance de l'éducation populaire : "L'éducation du peuple me paraît la plus saine cause de ce jour. Éducation, non instruction. Éducation morale, esthétique. Lectures de la Bible, des livres Indiens, de Michelet, de Lamennais, chants de psaumes, conversations ", écrivait-il en mai $1890^{\circ}$. Dès cette époque, avant même d'avoir quitté le lycée il était persuadé de la possibilité de mener une action sociale réformatrice, rejetant fermement l'action politique : «Je ne veux plus l'action politique : je nie le suffrage universel [...] Le suffrage universel, c'est la consécration du droit du plus fort ${ }^{10}$ ".

Au cours d'une période militaire en 1896, Daniel Halévy fit la connaissance déterminante de Paul Lagarde (1874-1908) avec lequel il se lia d'amitié. Cet avocat, qui avait épousé la fille de Paul Brousse, était l'administrateur du Prolétaire, organe de la Fédération des travailleurs socialistes de France. En outre, il était le secrétaire de Maurice Barrès qui l'avait chargé de diverses tâches à la Cocarde. Socialiste et anticlérical, plus tard franc-maçon, Lagarde était un militant particulièrement actif qui acquit une réputation d'avocat "avancé » au barreau de Paris, aux côtés de son ami Panayotis Argyriadès (1849-1901). Lagarde permit à Halévy de rencontrer Benoît Malon et l'introduisit auprès des réunions hebdomadaires de la Revue socialiste. Par ailleurs, il le présenta à Paul Roederer qui animait dans le quartier de la Villette un cercle protestant de rencontres entre étudiants et ouvriers. C'est dans ce cadre de la Société d'aide fraternelle et d'études sociales qu'Halévy fit ses premières expériences d'action sociale. Ce groupement avait été créé en 1882 par le pasteur Tommy Fal-

9. Ibid., 19 mai 1890.

10. Ibid., janvier 1891. 
lot, le fondateur du christianisme social en France ${ }^{11}$, qui avait été profondément influencé par la pensée de Charles Secrétan. Cette tendance minoritaire du protestantisme qui souhaitait rompre avec la philanthropie et les réformateurs sociaux issus de ses rangs, prônait une réforme des rapports entre capital et travail par le biais du coopératisme prôné par l'école de Nîmes et par l'éducation ouvrière. D. Halévy participa ainsi aux séances du groupe de la Villette à partir de la fin de l'année 1896. L'intérêt qu'il portait aux questions ouvrières par le biais de l'éducation populaire coïncidait avec l'irruption du mouvement ouvrier dans l'espace public. Les élections municipales de 1892, les législatives de 1893 ainsi que les élections municipales trois ans plus tard, marquées par la conquête de Lille et de Dijon, furent les premières étapes de l'ascension politique des formations socialistes.

\section{Libéralisme et socialisme à l'époque de l'affaire Dreyfus}

Le surgissement de l'affaire Dreyfus amena une évolution décisive dans la formulation de la pensée sociale et politique de Daniel Halévy. La famille Halévy fut enrôlée dans le camp dreyfusard à l'automne 1897 par l'action déterminante de Lucien Herr, secrétaire de rédaction de la Revue de Paris dont Ludovic Halévy était un des administrateurs et un des principaux actionnaires. Convaincus par Herr de l'irrégularité du procès et de l'innocence du capitaine Dreyfus dès la campagne de presse de Scheurer-Kestner en novembre 1897, les deux frères Halévy mobilisèrent leurs relations au service de la campagne révisionniste. Élie rassembla les camarades de la Revue de métaphysique et de morale et Daniel ceux du Banquet. Militant dreyfusard particulièrement actif, Daniel soutint en décembre 1897 le projet d'une adresse à Zola, qui ne vit finalement pas le jour, et recruta des signataires pour l'adresse de protestation de l'Aurore en janvier 1898, notamment Paul Lagarde et Paul Roederer. Il souscrivit à la médaille offerte par le Siècle à Zola en mars 1898 et signa l'Hommage des artistes à Picquart quelques mois plus tard. En novembre 1897, il ouvrit dans son

11. Cf. Jean Baubérot, «Le christianisme social français de 1882 à 1940 : évaluation et problème ", Revue d'histoire et de philosophie religieuse, vol. LXVII, 1, 1987, p. 37-63. 
Journal un dossier «Justice et passion » consacré à la chronique de l'Affaire, qui indiquait la double conviction de son engagement. Il entendait, comme la majorité des dreyfusards révisionnistes, prendre position contre une injustice mais aussi lutter contre les passions de l'opinion publique qu'il assimilait à la démagogie. Combat éthique d'une part, lutte antidémagogique de l'autre, celle-ci étant perçue dans sa dimension politique autant que culturelle. Les notes de «Justice et passion » montrent très nettement que des deux convictions, la seconde l'emportait sur l'engagement éthique. Cela signifiait pour lui qu'il fallait d'une part s'opposer aux démagogues, c'est-à-dire à la classe politique et à la grande presse, majoritairement et violemment antidreyfusarde, et d'autre part s'adresser à l'opinion, en l'occurrence les masses qu'il fallait éduquer. À la différence de bien d'autres dreyfusards pour qui le combat révisionniste favorisa un processus de socialisation politique républicain, l'engagement de Daniel Halévy ne se traduisit pas par un renforcement du sentiment d'attachement au régime. Bien au contraire, le comportement des masses nationalistes conforta chez lui une forme d'élitisme, l'attitude de la classe politique à l'égard des «passions » lui fournissant de nouveaux arguments contre le suffrage universel.

Tout au long de l'Affaire et pendant les années qui suivirent, Daniel Halévy mena un double engagement, libéral et socialiste. Il milita en premier lieu dans les rangs des libéraux révisionnistes. À l'invitation de Louis Havet, il participa dès la fin du mois de février 1898 à la collecte des soutiens pour la fondation de la Ligue des droits de l'homme ( $\mathrm{LDH})$. Aux origines la Ligue, organe non politique, s'inscrivait dans une démarche exclusivement « juridique et légaliste » ${ }^{12}$, recrutant majoritairement ses adhérents et ses cadres dans la bourgeoisie libérale ${ }^{13}$. Les liens des Halévy avec les premiers ligueurs à l'époque de la fondation étaient étroits. Gabriel Trarieux, le fils de Ludovic - premier président de la LDH - avait participé au

12. Emmanuel Naquet, "La Ligue des Droits de l'Homme au tournant du siècle", in L'Affaire Dreyfus et le tournant du siècle, 1894-1910, Nanterre, Musée d'histoire contemporaine-BDIC, 1994, p. 167.

13. Cf. l'étude prosopographique d'Emmanuel Naquet: «Les ligueurs des droits de l'homme dans le Maitron, de l'Affaire Dreyfus à la Seconde Guerre mondiale", Cahiers de l'IHTP, 26, mars 1994, p. 232-245. 
Banquet et Louis Havet, vice-président de la Ligue, était un ami de Ludovic Halévy. Par ailleurs, ils étaient également liés avec le second vice-président Émile Duclaux (1840-1904), par l'intermédiaire de leur amie Mary Darmesteter. Daniel Halévy continua à militer après l'Affaire à la section des Ternes de la Ligue, défendant auprès de Joseph Reinach la cause de Brière et celle de la présomption d'innocence. Par ailleurs, il milita également à l'Union pour l'action morale fondée par Jules Lagneau et Paul Desjardins en $1892^{14}$. Il avait adhéré avant l'Affaire à ce groupement dont certains membres fondateurs, Raoul Allier (1862-1939) et le pasteur Charles Wagner (18521939) notamment, faisaient partie de la Société d'aide fraternelle et d'études sociales de Tommy Fallot. "Association de mutuelle éducation philosophique et civique »d'après les statuts, l'Union avait pour objet d'aider à la recherche de la vérité et à la lutte pour le droit en mettant en œuvre les « méthodes critiques ». Daniel Halévy, après avoir protesté en décembre 1897 contre la neutralité de l'Union dans l'Affaire, devint membre du comité de rédaction du Bulletin de l'Union en novembre 1899.

Après avoir découvert le milieu broussiste de Paul Lagarde, Daniel Halévy fit la connaissance de deux représentants de la fédération socialiste de Seine-et-Oise et s'engagea alors à l'Union socialiste des trois cantons de Versailles, groupement allemaniste adhérent à la fédération. Il y rencontra Marius Lucas, tailleur de son état, secrétaire de la Bourse du travail et conseiller prud'homme à Versailles. À propos de cette rencontre, il écrivait : "Cela permet à des non-doctrinaires tels que moi d'entrer dans la vie socialiste, et d'y chercher, aidé par ces ouvriers intelligents sans la connaissance desquels toute pensée sur la société moderne est abstraite et vaine ${ }^{15}{ }^{»}$. Il se lia également avec Jean-Marie Jouandanne, magasinier dans une compagnie de chemins de fer, membre du groupe voisin de Port-Marly et secrétaire général de la fédération. Halévy assurait, notamment auprès de Jouandanne, un rôle de conseil pour l'organisation du mouvement dans le département. Il était particulièrement sollicité pour les questions

14. Cf. François Beilecke, «Die Union pour l'Action morale und die Union pour la Vérité : Zur Entwicklung und Rolle einer republikanischen Intellektuellenvereinigung 1892-1939 », Lendemains, 7879 , janvier 1995, p. 89-121. Nous renvoyons également à la contribution de François Chaubet dans ce numéro.

15. APH, Journal, 23 janvier 1899. 
d'éducation populaire. Il revint enthousiasmé de sa participation au congrès de la salle Japy. Muni des «instructions » ${ }^{16} \mathrm{de}$ Jouandanne, il représenta officiellement l'Union socialiste des trois cantons au congrès de la salle Wagram, mais revint écœuré - comme Charles Péguy un an plus tôt après Japy - par la violence des querelles intestines.

En 1901, Daniel Halévy publia son premier ouvrage, Essais sur le mouvement ouvrier en France, chez Lucien Herr à la SNLE. Ce livre très touffu est un document particulièrement intéressant pour analyser sa pensée sociale peu de temps après l'affaire Dreyfus. Il distinguait quatre formes d'actions du mouvement ouvrier auxquelles correspondaient quatre formes d'organisations, tour à tour étudiées: les syndicats et les bourses du travail, les institutions coopératives, les universités populaires ainsi que le parti socialiste. Halévy se livrait d'abord à une longue défense du syndicat et des bourses du travail, réfutant une à une toutes les critiques bourgeoises qui leur étaient opposées. Il renversait les arguments en affirmant qu'il était de l'intérêt du pouvoir d'encourager les syndicats car ils permettaient de canaliser la violence révolutionnaire. Le principe de la coopération était ensuite longuement défendu. Il voyait dans les coopératives de consommation et de production un moyen d'assurer la viabilité économique d'autres institutions ouvrières, notamment les UP. Mais surtout, il attachait une grande importance à la dimension «morale » de la coopérative : celle-ci modifiait le comportement de l'ouvrier, permettant à terme l'édification d'une contre-société où les masses ouvrières auraient acquis leur autonomie. En revanche, il déclarait son hostilité au principe de la grève générale et affirmait la nécessité d'un arbitrage de l'État dans les conflits du travail. Dans l'ensemble, l'ouvrage était l'apologie d'un projet révolutionnaire - l'autonomie ouvrière -, très fortement tempéré par l'attachement au principe coopératif, dimension perçue par les socialistes et les syndicalistes français comme réformiste ${ }^{17}$. En définitive, deux influences se mêlaient, celle du syndicalisme révolutionnaire et celle d'un christianisme

\footnotetext{
16. APH, lettre de J.-M. Jouandanne à D. Halévy, 10 septembre 1900.

17. Cf. Jean Gaumont, Histoire générale de la coopération en France. Formation et développement de l'Institution coopérative moderne, Paris, Fédération nationale des coopératives de consommation, t. II, 1923.
} 
social revu par l'école de Nîmes. D'un point de vue doctrinal, il faisait l'éloge de Malon, de Bernstein ainsi que de Proudhon dont l'influence bénéfique s'était traduite selon lui, par le désintérêt des socialistes français à l'égard de l'État. Ouvrage écrit après la participation au congrès de Wagram, les Essais marquaient très nettement son hostilité au socialisme de parti. Il critiquait l'organisation du parti socialiste et mettait en cause son existence même, assurant que l'intérêt du mouvement ouvrier devait l'entraîner à s'organiser de façon autonome, en dehors du suffrage universel. Lors de la publication de l'ouvrage, D. Halévy avait insisté pour que le titre comporte l'expression «mouvement ouvrier» et non «mouvement socialiste » comme le souhaitait Lucien Herr. Après la publication des Essais, Halévy abandonna toute activité militante à la fédération de Seine-et-Oise et il quitta définitivement les organisations socialistes. À Paris, d'autres causes l'avaient appelé.

\section{Le «deuxième dreyfusisme »}

À la suite de l'Affaire, s'ouvrit pour Daniel Halévy la période $\mathrm{du}$ «deuxième dreyfusisme » 18 qui l'amena à rejoindre ce qu'il nommait, à la suite de Proudhon, le «parti de la Démopédie ${ }^{19} »$. Georges Deherme (1867-1937), prenant exemple sur les soirées ouvrières organisées à Montreuil par Émile Méreaux, avait décidé de créer en 1898 un groupe d'éducation populaire à Paris ${ }^{20}$. Il avait largement utilisé le réseau de l'Union pour l'action morale dont il avait été un des fondateurs et sollicita ainsi Daniel Halévy pour qu'il effectue diverses conférences. Celui-ci fut donc un des premiers conférenciers de la Coopération des idées où il renoua avec l'expérience du groupe de la Villette. Deherme associa Halévy aux discussions préparatoires à la naissance de la Société des Universités populaires dont il devint un des administrateurs. Il fit la connaissance dans cette organisation de Maurice Kahn, de Charles

18. Daniel Halévy, Apologie pour notre passé, Cahiers de la Quinzaine, XI-10, 5 avril 1910 , p. 74 .

19. Daniel Halévy, Essais sur le mouvement ouvrier en France, Paris, SNLE, 1901, p. 154.

20. Cf. Lucien Mercier, Les Universités populaires, 1899-1914. Éducation populaire et mouvement ouvrier au début du siècle, Paris, Ed. ouvrières, 1986. 
Guieysse et de Marie Baertschi. À la fin de l'année 1899, il décida de répondre à un appel lancé par quelques employés désirant fonder une Université populaire. L'Enseignement mutuel, deuxième université populaire créée à Paris, fut ainsi fondée par Daniel Halévy, André Spire et Maxime Leroy qui s'installèrent rue de la Chapelle, non loin du quartier où Halévy avait fait ses premières expériences sociales auprès du pasteur Fallot. Le public de cette Université populaire, majoritairement composé d'employés et d'artisans, comprenait en réalité très peu d'ouvriers ${ }^{21}$. À la différence de Charles Guieysse et de Charles Péguy qui défendaient une conception ouvriériste de l'Université populaire qui devait être rattachée à un syndicat et exclure les éléments bourgeois ${ }^{22}$, Daniel Halévy concevait une organisation autonome diffusant la culture bourgeoise ${ }^{23}$. Cette conception assez répandue des UP postulait que la rencontre entre la culture libérale et l'humanisme démocratique était possible. Halévy y crut fermement, jusqu'à l'essoufflement du mouvement. Pour les bourgeois comme lui qui s'étaient lancés de façon précoce dans le mouvement des Universités populaires et qui occupaient des responsabilités importantes, l'avenir des UP était un indicateur important de la fonction culturelle et sociale des intellectuels dans la société.

Charles Guieysse avait proposé en 1900 à Daniel Halévy de s'associer au projet des Pages libres et celui-ci signa la «courte déclaration » du premier numéro. Péguy dont les Cahiers de la Quinzaine voisinaient avec les Pages libres a donné une juste définition du projet initial de cette revue : «Comme les Universités populaires, Pages libres font de l'enseignement primaire en ce sens que la considération des lecteurs, la formation des élèves y passe au premier plan ${ }^{24} \gg$. Aux Pages libres, Halévy remplissait les mêmes fonctions qu'à la fédération de Seine-et-Oise : il fut un conseiller permanent de Guieysse et assurait, à sa demande, la direction de certains numéros. Il

21. Cf. "L'enseignement mutuel ", Bulletin des Universités populaires, 2, juin-juillet 1900, p. 20.

22. Cf. Charles Guieysse, Les Universités populaires et le mouvement ouvrier, Cahiers de la Quinzaine, III-2, octobre 1901, avec une préface de Charles Péguy, "Vraiment vrai ".

23. Daniel Halévy, "Opinions sur les U.P.", Pages libres, 191, 27 août 1904 , p. 161-167.

24. Ch. Péguy, "Vraiment vrai ", loc. cit., p. 8. 
concevait la revue comme une institution « libérale » ${ }^{25}$ dont la tâche d'éducation devait notamment limiter les effets de l'humanitarisme démocratique. Pierre Monatte qui travailla un temps à la revue observait :

Quand je pense à ce milieu de Pages libres [...] je suis obligé de me rendre compte que je le regardais, sinon d'un peu haut, au moins comme ne cadrant pas complètement avec mes propres convictions. On y avait le sens de la liberté, mais on ne s'y proclamait pas libertaire [...] On n'était donc pas à Pages libres assez libertaire pour mon goût ${ }^{26}$.

Les contributions de Daniel Halévy à la revue illustrent la position particulière qu'il occupait à l'égard du mouvement ouvrier. Il ne publia pas un seul texte à caractère politique et ne se prononça pas sur les grandes questions du temps telles que la loi de séparation, l'hervéisme ou les lois sociales qui emplissaient pourtant les pages de la revue. Les questions culturelles le préoccupaient plus et il rédigeait des notes de lecture sur des ouvrages littéraires ou sociaux susceptibles d'intéresser le lectorat des Pages libres. Il mena surtout à partir de 1901 une vaste «enquête ouvrière »: il rédigea ainsi au fil des numéros une série de portraits sur les grandes figures françaises et anglaises du mouvement ouvrier, chartiste et républicain, rencontrant les survivants et interrogeant leurs proches. Par ailleurs, il publia une série d'études sur les grands épisodes de la lutte ouvrière et parcourut la France, utilisant le réseau des abonnés pour enquêter sur les organisations et la vie ouvrières.

Le socialisme de Daniel Halévy présentait de nombreux points communs avec celui de Péguy ${ }^{27}$. À l'origine de leur démarche, il y avait une interrogation analogue d'essence morale, kantienne chez Péguy, issue du protestantisme chez Halévy. Tous deux avaient également admiré Jaurès. Ils quittèrent le parti socialiste presque à la même époque et dans des circonstances identiques. Évoquant Péguy en 1909, Daniel Halévy parlait aussi pour lui-même : «Il n'était pas né pour

25. Daniel Halévy, "Préface " à Charles Guieysse, Pages libres. Extraits, Hennebont, Impr. J. Méhat, 1927, p. 5.

p. 11 .

26. Pierre Monatte, "Paris 1902 ", Témoins, 8, printemps 1955,

27. Cf. Géraldi Leroy, Péguy entre l'ordre et la révolution, Paris, Presses de la FNSP, 1981. 
suivre un état-major de fonctionnaires enseignant la révolution par doctrine, ni pour s'accorder avec ces masses dont il avait tant espéré, et qu'il voyait obéissantes à des guides si médiocres ${ }^{28}$ ». Halévy enfin paraissait souscrire à la dimension libertaire du socialisme de Péguy. Dans un article de 1909 des Pages libres consacré aux nouveaux aspects du socialisme, il revenait sur une conférence faite par Péguy à l'École des hautes études sociales en janvier 1904 dans laquelle celui-ci s'était penché sur la question du pouvoir et de l'autorité :

Il avait défini deux formes de l'autorité, la forme cratie et la forme archie ; celle-ci noble, celle-là dégradée. La cratie, disait-il, est l'autorité sans passé, sans principe et qui s'impose par la peur. L'archie est fondée sur des supériorités réelles, sur de longues expériences, sur des croyances, des nuances de consentement intime, des adhésions loyales [...] Il se déclarait lui-même acratiste, mais archiste. Acceptons cette terminologie: elle a du sens, comme tout ce qu'écrit Charles Péguy ${ }^{29}$.

En 1943, Daniel Halévy reviendra sur cette nuance dans une lettre adressée à Charles Maurras : «La difficulté relative à l'opposition verbale hiérarchiste-anarchiste peut être levée. Les jeunes Idéophages ne se considéraient pas comme des anarchistes mais comme des libertaires et Péguy, très sensiblement libertaire, a fait une distinction précieuse entre les archies et les craties. Mes amis étaient acrates, non anarchistes. Et moi aussi, j'étais acrate ${ }^{30}$ ! » Enfin, une méfiance profonde chez Péguy ${ }^{31}$ pour le suffrage universel et pour le parlementarisme, prenant la forme d'une véritable hostilité chez Halévy, rapprochait les deux hommes. Grâce à Péguy qui lui ouvrit les Cahiers de la Quinzaine, Daniel Halévy put reprendre et

28. Daniel Halévy, "Les Cahiers de Charles Péguy », le Temps, 12 décembre 1909 , p. 3.

29. Daniel Halévy, "Les nouveaux aspects du socialisme ", Pages libres, 457, 2 octobre 1909, p. 371. La sténographie complète de cette conférence a été publiée: "Une conférence sur l'anarchisme politique", Feuillets mensuels de l'Amitié Charles Péguy, 139 et 140, 15 mars, 15 avril 1968, p. 3-36 et 2-28.

30. Archives privées Paul Beauvais, lettre de D. Halévy à Charles Maurras, 25 mai 1943. 139.

31. G. Leroy, Péguy entre l'ordre et la révolution, op. cit., p. 138- 
amplifier en 1903 sa carrière littéraire, interrompue de façon précoce par l'affaire Dreyfus et le «deuxième dreyfusisme ».

Les premières mentions d'Halévy dans les documents connus de Sorel datent de 1905 et le nom de Sorel apparaît pour la première fois dans les papiers d'Halévy en 1907. En fait, les deux hommes se connaissaient depuis quelques années et Halévy témoigna de ce point de vue d'une bonne connaissance de l'œuvre de Sorel, lorsqu'il distingua dans les articles du Mouvement socialiste parus en 1906 une pensée forte et originale pouvant constituer la matière d'un livre - les Réflexions sur la violence - qu'il éditera avec André Spire à la librairie des Pages libres. L'élitisme, l'ouvriérisme et la condamnation de la démocratie parlementaire par Sorel étaient des traits qui le rapprochaient de Daniel Halévy. Mais celui-ci distinguait l'homme de l'œuvre : son attitude à l'égard des Réflexions est emblématique de sa faculté à s'intéresser aux idées neuves, tout en se tenant à distance d'elles. Ce livre qui était un hymne à la gloire du mouvement ouvrier était également une apologie du mythe de la grève générale, un éloge de la violence prolétarienne et un des textes fondateurs de l'anti-intellectualisme. Cela n'empêchait pas Halévy d'en parler comme « le plus beau livre de morale, le plus beau traité sur les mœurs qui ait été écrit depuis La Justice de Proudhon », mais il ajoutait : «Comprenons l'auteur, n'oublions pas les différences ${ }^{32} \gg$. Ainsi, il n'abdiquait pas devant celui-ci de son jugement critique et il écrivait dans ses Notes :

Il est nécessaire d'écouter ce vieux maître, qui est précieux par la dignité de sa vie, son impatience des formules [...] et la violence de sa pensée. Mais il est nécessaire d'oser ne le point suivre. Comme Ulysse, s'attacher au mât de son navire, pour entendre, s'instruire et passer, comme Ulysse dédaigner de s'étouper les oreilles [...] Il est bon de promener ses pensées dans la rudesse des vents contraires qui les froisse, dérange leurs commodes allures ${ }^{33}$.

\section{La décomposition du « deuxième dreyfusisme »}

L'idée de révolution ne cessait de faire des progrès dans les rangs du syndicalisme : au début du siècle, le mouvement

32. APH, Notes $X, \S 72$, [ca. début 1909].

33. Ibid., § 22, [ca. mars-mai 1907]. 
ouvrier était à l'apogée de sa puissance et de sa capacité de mobilisation. C'est dans ce contexte que put éclore un messianisme ouvrier, notamment dans les rangs du syndicalisme révolutionnaire qui portait une revendication primordiale, celle de la grève générale. Après la mort de Pelloutier en 1901, l'emprise croissante de la CGT (qui avait absorbé les Bourses du travail en 1902) sur le mouvement syndical, se fit de plus en plus forte. Daniel Halévy était très hostile au caractère centralisateur de la CGT, au peu de souci qu'elle avait dans la pratique des questions d'éducation, à certains modes d'action qui lui répugnaient. Ses Notes de 1909 résument la distance qui le séparait de la nouvelle classe ouvrière : «On ne travaille pas, on n'encourage pas le travail à la CGT. Point d'enseignement professionnel, point de mutualisme ni de coopérative - on subordonne aux préoccupations d'attaque brusque, de pillage et de destruction. Former des bandes pour le brigandage, non des hommes pour le travail ${ }^{34}{ }^{»}$. À l'aune de cette évolution, il analysait son engagement antérieur :

Il y a dix ans les syndiqués avaient un autre orgueil. Les patrons ne peuvent pas nous chasser. Ils ont besoin de nous parce que nous sommes les meilleurs ouvriers, disaient-ils souvent. J'ai souvent entendu de telles paroles. Maintenant... Non, en vérité : je pense à ces doctrines nouvelles, et à nos anciennes doctrines - il y a dix ans. Je ne vois pas que nous devrions changer. Que disions-nous il y a dix ans? Quatre rapides années nous avaient instruits. Nous disions qu'il était nécessaire que la classe ouvrière se crée les éléments, les instruments, les institutions d'une culture. Nous : je dis des P[ages] L[ibres], aux Cahiers, et les U.P. et Sorel le disaient aussi. Le syndicalisme moderne déclame contre la démocratie: [nous déclamions] moins, mais nous étions moins qu'eux démocrates. Nous ne flattions aucune foule, nous venions d'être instruits par l'A[ffaire] $D$ [reyfus] de ce [que] valent les foules, et tout notre travail était dirigé contre elles. Par l'organisation, par les institutions, supprimer la foule [...] nous pensions avec B[ernstein] que le mouvement est tout, le but final rien; et que tout le travail de la classe ouvrière doit consister à développer ses institutions, ses mours et son idéal ${ }^{35}$.

34. Ibid., \& 72 [début 1909].

35. Ibid. 
L'affaire Dreyfus avait permis la rencontre des intellectuels et du monde ouvrier par le biais du mouvement socialiste et, marginalement, du syndicalisme. L'expérience des UP avait été une des entreprises communes résultant de cette rencontre. Les UP avaient cristallisé les critiques du monde ouvrier à l'égard des intellectuels, et les attaques redoublèrent lors de l'échec rapide du mouvement. Ainsi se forma autour de la représentation de l'intellectuel bourgeois éducateur une des figures les plus caractéristiques de l'anti-intellectualisme de gauche. L'échec des UP et l'exploitation politique de la mobilisation ouvrière en faveur de Dreyfus par les radicaux, précipitèrent vers 1907-1908 une évolution au cours de laquelle la méfiance céda rapidement la place à un anti-intellectualisme vigoureux s'exprimant souvent sur le mode pamphlétaire.

Tirant les leçons de l'évolution du mouvement ouvrier et de la désaffection croissante à l'égard de l'UP, l'Enseignement mutuel fut dissous en décembre 1906. Daniel Halévy, interrogé en 1930 par André Rousseaux, expliquait ainsi la fin de ce dernier : «Nous en avons été expulsés, moi et ceux qui, comme moi, avaient pris à cœur ces conférences et ces réunions, vers 1909. Le syndicalisme fit triompher alors les méthodes violentes; les bourgeois que nous étions furent rejetés. Je fus alors assez désemparé ${ }^{36} »$. Il publia alors aux Cahiers de la Quinzaine un court texte intitulé Un épisode, sur la fin dramatique d'une UP, une œuvre sombre qui était très largement autobiographique.

En octobre 1909, les Pages libres à court d'argent, disparaissaient. Aux dissensions nées de l'exploitation politique de l'Affaire, à la lente agonie du « deuxième dreyfusisme » caractérisée par la disparition des Pages libres et de l'Enseignement mutuel, s'ajoutèrent pour Halévy le retrait de la Ligue des droits de l'homme. Depuis l'Affaire, la Ligue avait nettement évolué dans le sens d'une politisation allant bien au-delà de la lutte originelle pour le respect du droit. Francis de Pressensé, président de la Ligue depuis 1903, appuya en effet largement le Bloc et la politique de Combes, puis défendit celui-ci lors de l'affaire des fiches. Cette attitude heurta de nombreux libéraux, provoquant une vague de démissions au sein de la LDH et des

36. André Rousseaux, «Un quart d'heure avec M. Daniel Halévy ", Candide, 17 avril 1930. Les erreurs de datation sont fréquentes dans les souvenirs d'Halévy. 
protestations de nombreuses sections ${ }^{37}$. Pressensé incarnait incontestablement un nouvel esprit. Après la victoire du Bloc, une nouvelle vague de militants adhéra à la $\mathrm{LDH}$ : plus politisés, moins liés à l'événement fondateur qu'avait été l'Affaire, ils contribuèrent à transformer la ligue libérale des premiers temps en une « société politique » ${ }^{38}$. Péguy qui n'adhérait plus à la Ligue depuis 1903, lui était devenu hostile : il décida de lui consacrer un cahier polémique en janvier 1905, La Délation aux Droits de l'homme, directement inspiré par l'affaire des fiches. Dans le texte précédant le dossier, il employa pour la première fois le terme de «décomposition du dreyfusisme », caractérisant le nouvel état d'esprit. Daniel Halévy réagit rapidement en lui écrivant le $1^{\text {er }}$ février 1905 : «Mon cher Péguy, pourquoi ne me racontez-vous pas vos affaires? Spire me dit que vous lui avez demandé son nom pour un cahier Bouglé sur la délation, lequel cahier comporterait des adhésions. Prenez le mien ${ }^{39}{ }^{\gg}$. La réaction d'Halévy témoigne d'un jugement convergent avec Péguy. Hostile aux changements de la LDH, il quitta la Ligue, probablement au moment de l'affaire des fiches : en tout état de cause, il n'en était plus membre en $1906^{40}$. Elle devint alors la cible de ses attaques, provoquant une polémique avec Maxime Leroy, un des juristes importants de la Ligue et un ancien camarade d'UP : «Qu'est devenue la Ligue des droits de l'homme dont Émile Duclaux fut d'abord vice-président? La foule de ses quatre-vingt mille adhérents l'abaisse, et le plus bas travail électoral est sa besogne », écrivait Halévy en mars $1907^{41}$. À cette époque l'isolement intellectuel d'Halévy était réel. Il écrivait ainsi au printemps 1908 à Georges Guy-Grand (1879-1957), qui dirigeait les Annales de la jeunesse läqque : « Je vous lis, quant à moi, parce que je me trouve dans une situation très pareille à la vôtre. Je crois fon-

37. Cf. Guy Thuillier, « Morale et politique : Péguy et l'affaire des "fiches" en 1905 ", Revue administrative, 256, juillet-août 1990, p. 310-316.

38. Emmanuel Naquet, "La Ligue des droits de l'homme : une politique du droit et de la justice dans le premier vingtième siècle ", Jean Jaurès cahiers trimestriels, 141, juillet-septembre 1996, p. 36.

39. Archives du Centre Charles Péguy, CORCQ-IV-39, inv. 02214,

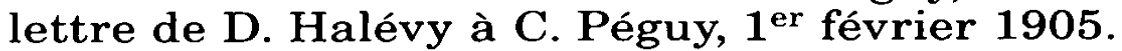

40. D'après les indications du Bulletin officiel de la Ligue des droits de l'homme.

41. Daniel Halévy, «Émile Duclaux (1840-1904)», Pages libres, 322, 2 mars 1907, p. 228. 
dées presque toutes les critiques qu'on adresse à notre démocratie. Je ne me sens pas en état d'avaler la médecine réactionnaire. D'où une sérieuse difficulté de vivre et un état peu agréable où j'ai plaisir à ne pas me sentir seul ${ }^{42}$. »

En avril 1910, Daniel Halévy publia chez Péguy un témoignage sur l'affaire Dreyfus, l'Apologie pour notre passé. L'Apologie est non seulement une analyse de son engagement personnel mais également du comportement des libéraux et des orléanistes lors de l'Affaire. Halévy faisait dans son cahier un examen particulièrement critique de ces milieux tout en écrivant de très pertinentes et rares pages d'une " histoire des libéraux conservateurs dans l'Affaire Dreyfus». Un regret transparaît de façon très nette tout au long de l'Apologie quant au comportement de ceux qu'il tenait pour ses « guides » naturels. «Je pense à un groupe de très sages Français que nous avions l'habitude d'écouter avec attention ", écrit-il ${ }^{43}$. Et il évoquait Gaston Paris, Émile Faguet, le vicomte de Vogüé, Sully-Prudhomme, le comte d'Haussonville, le duc de Broglie, Émile Boutroux, Anatole Leroy-Beaulieu : «Ils continuaient assez bien parmi nous cette manière de penser qu'on appelait, voici quelque trente ans, l'orléanisme ${ }^{44} \gg$. Il regrettait qu'aucun d'entre eux n'ait fait entendre sa voix à l'époque : "Continuant une tradition si sûre, nous aurions pris garde à leurs conseils [...] Mais ils n'en donnaient pas. De vagues propos nous laissaient deviner les dispositions humaines communes à tous, qui menaient la plupart d'entre eux jusqu'au désir d'une révision, et qui mettaient les autres dans un état pénible de doute, d'embarras ${ }^{45}$ ». En fait, le camp orléaniste s'était scindé en deux à la fin de l'année 1898 et au début de l'année 1899 , entre d'une part ceux qui avaient adhéré à la Ligue de la patrie française tels Broglie, Vogüé, Haussonville, Faguet et d'autre part ceux qui signèrent l' «appel à l'union » du Temps. Parmi les hommes qu'Halévy avait cités, Gaston Paris, Boutroux, Sully-Prudhomme le signèrent (ainsi que son père, non mentionné dans l'Apologie). Le regard porté par Halévy sur ces personnalités est révélateur d'un certain malaise ressenti par lui au sein de la coalition dreyfusarde. Il exprimait ainsi le

42. Archives privées Guy-Grand, lettre de D. Halévy à Georges Guy-Grand, 8 mars 1908.

43. D. Halévy, Apologie pour notre passé, loc. cit., p. 54.

44. Ibid., p. 54-55.

45. Ibid., p. 55. 
paradoxe de sa situation : «Pourquoi ne nous aidiez-vous pas, ou si peu, si mal et si loin, nous laissant insulter, nous insultant parfois, vous qui dans le fond n'étiez pas loin de nous, plus près en tout cas, infiniment plus près que tels autres qui nous aidèrent ${ }^{46}$ ». Dans cette situation: "Nous suivions enfin les guides qui s' offraient : un Zola, un France, un Duclaux, un Pressensé, un Louis Havet ${ }^{47} \gg$. L'intérêt de l'Apologie est de montrer en fin de compte, dans l'analyse du comportement des libéraux, les nuances de l'engagement d'Halévy en faveur de Dreyfus. Il manifestait à la fois un attachement incontestable à la cause dreyfusarde et en même temps le profond regret que les orléanistes n'aient pas conservé le rôle qui était le leur : rester des «guides » pour la société libérale dont Halévy faisait partie. Il n'avait dès lors plus d'autre choix que celui de suivre une autre tradition : "Car l'ensemble des émotions, des solidarités républicaines constitue très exactement une tradition, la dernière qui nous reste ${ }^{48} \gg$. Ainsi Daniel Halévy étaitil mal à l'aise dans le manichéisme créé par l'Affaire. L'Apologie montre clairement son regret de n'avoir pu défendre à la fois la cause de Dreyfus et certains principes conservateurs : Halévy, à la différence de nombre de ses compagnons dreyfusards, rappelait qu'il ne ressentait aucune hostilité de principe envers l'institution militaire ou vis-à-vis de la magistrature. Son histoire des libéraux, qui constitue à bien des égards leur procès, s'achève par un verdict sévère qui révèle toutes les ambiguïtés de sa propre position : «L'instinct conservateur a été faible en nous, soit ! Était-ce donc à nous, libéraux par choix, tradition, goût, à l'incarner ? C'est à vous que cette tradition était commise, hommes de la droite ; vous deviez la défendre par vos actes, l'honorer par vos vies. Vous vous êtes déshonorés, vous l'avez déshonorée ${ }^{49}$ ».

Il est intéressant de noter la façon dont il caractérisait en 1910 son propre engagement d'antan. C'est par touches successives qu'il l'évoquait, se disant à la fois libéral, « démopédiste, non-démocrate » et républicain, mais d'une façon très particulière : «Notre républicanisme [...] ignorait cet organisme central, l'État, autoritaire par nécessité, et s'intéressait uniquement aux organismes moins visibles, non moins visi-

46. Ibid., p. 62 .

47. Ibid., p. 64 .

48. Ibid., p. 82 .

49. Ibid., p. 110. 
bles, où l'élite des peuples s'entraîne par un exercice quotidien aux disciplines de la vie civique, et protège ses libertés 50 ». L'élitisme de son engagement était clair : «La fin que nous poursuivons, qui est, si nous ne nous trompons, la culture des qualités humaines, le maintien d'un certain goût et d'un certain honneur, n'est pas de celles où concourent les forces fondamentales ${ }^{51} \gg$. En définitive, il savait parfaitement que son attitude le menait vers une certaine marginalisation au sein de l'ancienne cohorte dreyfusarde : «Recommençons souvent la critique de nos amis, révisons nos traités d'alliance. Soucionsnous de notre liberté, dussions-nous être par ce souci condamnés à vivre un peu retirés, un peu confinés 52 ".

L'ouvrage provoqua la réplique cinglante de Péguy, Notre jeunesse et une brouille entre les deux hommes. Bien que Péguy ait atténué par la suite ses propos à l'égard d'Halévy dans Victor-Marie comte Hugo, celui-ci quitta définitivement les Cahiers. La seule attache qui retenait désormais Halévy à son passé dreyfusard était l'Union pour l'action morale. Devenu membre du Conseil d'administration de l'Union en 1906, Halévy devint secrétaire du bulletin de l'Union en 1910 à la demande de Paul Desjardins. Mais, à la suite d'un différend avec Desjardins, il quitta l'Union deux ans plus tard.

Du dreyfusisme et du "deuxième dreyfusisme », il ne restait plus rien d'autre pour Daniel Halévy que des convictions personnelles. Un à un, tous les groupements nés de l'Affaire avaient disparu. Halévy, pourtant, ne remit jamais en cause par la suite la justesse de son combat révisionniste. La pensée sociale syncrétique qui l'avait amené au socialisme avait été marquée à l'origine par une dimension morale et idéaliste. Celle-ci fut durable et c'est probablement une des raisons pour laquelle l'engagement au parti socialiste fut très bref. Il reste que c'est dans les Universités populaires et aux Pages libres qu'il trouva un esprit conforme à sa culture libérale et à ses aspirations sociales. Après 1910-1912, toutes les attaches avec les milieux républicains et socialisants ayant été rompues, la dimension conservatrice de son libéralisme l'emporta de plus en plus.

50. Ibid., p. 86 .

51. Ibid., p. 101.

52. Ibid. 